\title{
Strengthening of Adhesive Bonding Structure Using Energy-Release-Rate Reduction Slit*
}

\author{
Shinsuke TAKeUCHI, ${ }^{1)}$ Eiichi SATO, ${ }^{1)}$ Nobuaki KAWAI ${ }^{1,2)}$ and Iori NAKAMURA ${ }^{1), 3)}$ \\ ${ }^{1)}$ Institute of Space and Astronautical Science, Japan Aerospace Exploration Agency, Kanagawa 252-5210, Japan \\ ${ }^{2)}$ Present Address; Institute of Pulsed Power Science, Kumamoto University, Kumamoto 860-0862, Japan \\ ${ }^{3)}$ Present Address; SHARP Corporation, Nara 632-8567, Japan
}

\begin{abstract}
A new method of strengthening adhesive bonding structures, introducing a slit near the bonding layer, is proposed in order to solve a problem affecting composite pressure vessels; that is, delamination of the bonding layer between the composite and metal, especially around a mouthpiece. The effect of the slit is studied on the basis of an analytical solution, and evaluated using actual double cantilever beam experiments and finite element method calculations. An example of composite pressure vessel design is presented to illustrate the effect of the slit.
\end{abstract}

Key Words: Bonding Structure, Fracture Mechanics, Energy Release Rate

\section{Nomenclature}

a: crack length, $\mathrm{m}$

$B$ : breadth of DCB test piece, $\mathrm{m}$

$C$ : compliance with slit, $\mathrm{m} / \mathrm{N}$

$C_{0}$ : compliance without slit, $\mathrm{m} / \mathrm{N}$

$E$ : Young's modulus, $\mathrm{Pa}$

$F$ : force at the moment of crack growth in DCB with slit, $\mathrm{N}$

$F_{0}$ : force at the moment of crack growth in DCB without slit, $\mathrm{N}$

$J$ : energy-release rate with slit, $\mathrm{J} / \mathrm{m}^{2}$

$J_{0}$ : energy-release rate without slit, $\mathrm{J} / \mathrm{m}^{2}$

$J_{c}$ : critical energy-release rate of bonding layer, $\mathrm{J} / \mathrm{m}^{2}$

$K_{\mathrm{I}}$ : stress-intensity factor at pre-crack tip with slit, $\mathrm{Pa} \sqrt{\mathrm{m}}$

$K_{\mathrm{I} 0}$ : stress-intensity factor at pre-crack tip without slit, $\mathrm{Pa} \sqrt{\mathrm{m}}$

$K_{\mathrm{I}}^{\prime}$ : stress-intensity factor at diminutive internal crack tip with slit, $\mathrm{Pa} \sqrt{\mathrm{m}}$

$L_{0}$ : pre-crack length, $\mathrm{m}$

$M$ : membrane bending moment, $\mathrm{N}$

$T$ : membrane force, $\mathrm{N} / \mathrm{m}$

$a$ : half length of diminutive internal crack, $\mathrm{m}$

$l$ : slit length, $\mathrm{m}$

$r, r_{1}, r_{2}$ : see Fig. $2, \mathrm{~m}$

$r^{\prime}, r_{1}^{\prime}, r_{2}^{\prime}$ : see Fig. 7, m

$t$ : distance between slit and pre-crack, $\mathrm{m}$

$\beta$ : dimensionless stress-intensity factor

$\epsilon$ : elastic strain

$\kappa$ : curvature (inverse of bending curvature radius), $1 / \mathrm{m}$

$\theta, \theta_{1}, \theta_{2}:$ see Fig. 2, rad

(c) 2015 The Japan Society for Aeronautical and Space Sciences

*Received 30 June 2014; final revision received 29 November 2014; accepted for publication 17 December 2014. $\theta^{\prime}, \theta_{1}^{\prime}, \theta_{2}^{\prime}:$ see Fig. 7, rad

$\sigma$ : stress made by slit at pre-crack tip, $\mathrm{Pa}$

$\sigma^{\prime}$ : stress made by slit at center of diminutive internal crack, $\mathrm{Pa}$

$\sigma_{0}$ : uniform stress at infinite distance, $\mathrm{Pa}$

$\hat{*}$ : superscript ${ }^{\wedge}$ indicates a value after crack growth

$*_{P}$ : subscript ${ }_{P}$ indicates a value affected by pressure

\section{Background}

Lightweight composite vessels are expected to be replacements for traditional metal tanks. High-pressure helium gas tanks are used on many launch vehicles and satellites ordinarily. Moreover, high-pressure vessels for room-temperature ground use are also being used or considered for compressed natural gas $(\mathrm{CNG})$ automobiles, hydrogen automobiles and fuel-cell automobiles (As an example, see website of Lincoln Composites in the USA; http://www.lincolncomposites.com).

Launch vehicles use a cryogenic propellant. In particular, composite tanks for liquid hydrogen were developed for the DC-X and X-33 projects in the USA. These projects were cancelled due to landing failures ${ }^{1)}$ and leakage of the tanks through matrix micro-cracks, ${ }^{2)}$ respectively. NASA has continued developing composite tanks for liquid hydrogen ${ }^{3)}$ and very recently has carried out a series of tests on a cryogenic composite tank manufactured by Boeing. ${ }^{4)}$

Meanwhile the authors' group successfully developed cryogenic composite tanks using metal ${ }^{5)}$ and polymer ${ }^{6)}$ liners respectively during a reusable vehicle testing (RVT) project in Japan. These high-pressure cryogenic composite tanks require metal mouthpieces in order to seal external pipes with the current technology. This requires dissimilar materials to be bonded, and hence, delamination could become a critical constraint in the design process.

Especially for a cryogenic composite tank, delamination of the bonding may be serious problem because fracture toughness decreases at cryogenic temperatures. It has been 
shown that a design method based on fracture mechanics can solve this problem at the edge of mouthpieces. ${ }^{7)}$

Meanwhile, for pressure vessels at room-temperature, the stress or strain of the material is a normal design constraint. Additionally fatigue tolerability is verified. ${ }^{8)}$ However, Takeuchi et al. ${ }^{7)}$ pointed out that the energy release rate (ERR), not the stress or strain, will be the dominant factor in designing extremely high-pressure or huge tanks in the future. To see this, the ERR at the edge of a mouthpiece without thermal stress can be expressed as

$$
J_{P}=\frac{1}{2} T_{P}\left(\hat{\epsilon}_{P}-\epsilon_{P}\right)+\frac{1}{2} M_{P}\left(\hat{\kappa}_{P}-\kappa_{P}\right) .
$$

In this equation, the membrane force, membrane moment, strain and curvature are in proportion to the internal pressure; thus, the ERR is in proportion to the square of the internal pressure. On the other hand, stress and strain are only proportional to the first order of the internal pressure. Thus, at high pressure, ERR is more critical than either stress or strain.

Moreover, the above equation shows a kind of scale effect of fracture mechanics; that is, the ERR includes the dimension of length. Therefore, it increases as the size of the tank grows, whereas the stress or strain remains constant as long as the tanks are similar in shape. Thus, ERR is more important than stress or strain when designing huge tanks.

This paper describes a method of dealing with the predicted ERR problem and preventing fractures in future tanks. Although this problem potentially affects both high-pressure tanks at room-temperature and huge cryogenic tanks, in this paper we focus on high-pressure gas tanks at room-temperature because of the simplicity of the formulation. It has been found that the simple solution of making a slit in the mouthpiece can reduce ERR by an order of magnitude. ${ }^{9)}$ The effectiveness of this method is evaluated using an analytical approach, double cantilever beam (DCB) experiments conducted at room-temperature, and numerical simulations. A large high-pressure gas tank for room-temperature use is then designed to illustrate the effect of adding a slit.

\section{Problems with Composite Tanks}

\subsection{Mouthpiece bonding delamination}

Figure 1 shows an outline of two types of composite tank: (a) polymer-lined composite tank and (b) metal-lined composite tank. Takeuchi et al. ${ }^{7)}$ showed that one type of delamination between the composite and metal mouthpiece starts from the outer edge of the mouthpiece (part B in Fig. 1(a)) in polymer-lined composite tanks. It also shows that the ERR of delamination, or interfacial crack growth, can be reduced by shaving the edge of the mouthpiece. In particular, our previous tank was designed to have as thin a mouthpiece as possible. $^{6)}$

On the other hand, another type of delamination starts from the bonding boundary (part A in both Figs. 1(a) and (b)). This problem affects not only polymer-lined composite tanks, but also metal-lined composite tanks, which are more common at present. It was found during the development of

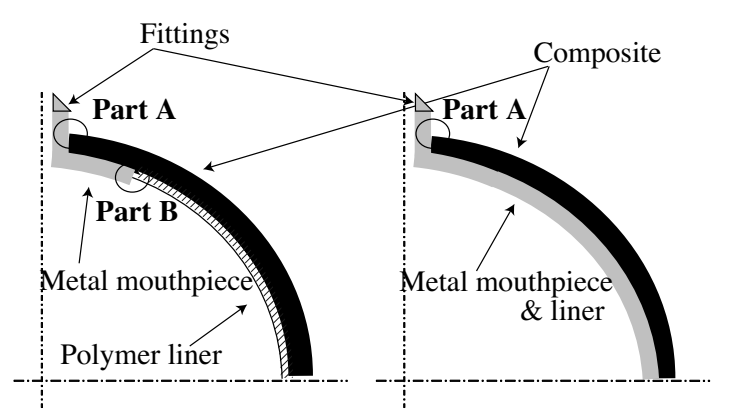

(a) Polymer-lined composite tank (b) Metal-lined composite tank

Fig. 1. Outline of two typical composite tanks.

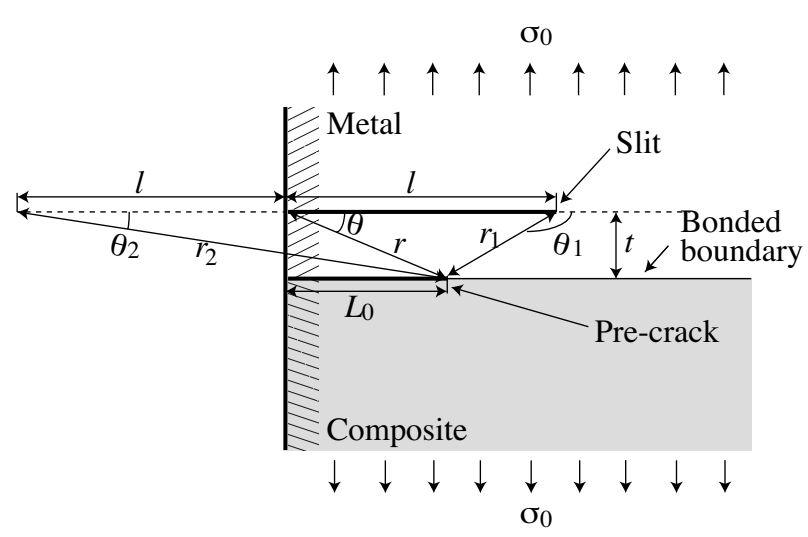

Fig. 2. Mathematical model of pre-crack and slit.

the metal-lined composite tank in Higuchi et al. ${ }^{5)}$ that delamination started from this part and resulted in liner breakage. This paper focuses on delamination from this part.

\subsection{Energy-release-rate reduction by slit}

Making a slit in the mouthpiece can reduce the ERR at the bonding part of the tank mouthpiece. The slit near the bonded boundary reduces the local rigidity of the metal mouthpiece, relaxes the stress at the tip of the pre-crack in the boundary, and thus reduces the ERR in the bonding part. Although the slit would seem to be a new pre-crack, the fracture toughness of metals is generally much higher than that of adhesives.

To simplify the problem, let us discuss a semi-infinite plate having uniform stiffness and a surface pre-crack in the plane strain state. Figure 2 shows the geometrical configuration around the pre-crack and the slit. A metal piece and a composite piece having the same stiffness are bonded at the center of the model, and there is a surface pre-crack whose length is $L_{0}$ along the bonded boundary. The slit in the metal piece is parallel to the boundary, its length is $l$, and the distance from the boundary is $t$.

When a stress at infinite distance $\sigma_{0}$ is uniformly applied and there is no slit, the stress intensity factor (SIF) at the pre-crack tip $K_{\mathrm{I} 0}$ can be expressed as

$$
K_{\mathrm{I} 0}=\beta \sigma_{0} \sqrt{\pi L_{0}},
$$

where $\beta$ equals 1.122 for a surface pre-crack. When there is no pre-crack, the stress due to the slit at the point of the pre- 


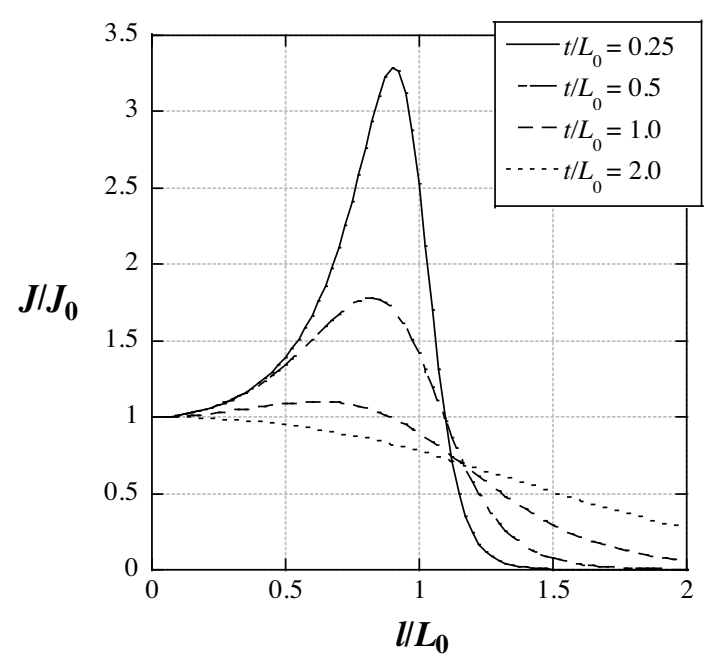

Fig. 3. Analytical results for energy release rate.

crack tip, $\sigma$, can be approximated as ${ }^{\dagger}$

$$
\begin{aligned}
\sigma= & \frac{\sigma_{0} r}{\sqrt{r_{1} r_{2}}}\left\{\cos \left(\theta-\frac{\theta_{1}+\theta_{2}}{2}\right)\right. \\
& \left.+\frac{l^{2}}{r_{1} r_{2}} \sin \theta \sin \frac{3}{2}\left(\theta_{1}+\theta_{2}\right)\right\},
\end{aligned}
$$

where the variables $r, r_{1}, r_{2}, \theta, \theta_{1}$ and $\theta_{2}$ are derived from the geometrical configuration in Fig. 2.

To estimate the SIF at the pre-crack tip when there is a slit, $K_{\mathrm{I}}$, let us suppose that the infinite stress $\sigma_{0}$ decreases to $\sigma$ and is given by Eq. (3). $K_{\mathrm{I}}$ is given by

$$
\begin{aligned}
K_{\mathrm{I}}= & \beta \sigma \sqrt{\pi L_{0}} \\
= & \frac{\sigma_{0} r \sqrt{\pi L_{0}}}{\sqrt{r_{1} r_{2}}}\left\{\cos \left(\theta-\frac{\theta_{1}+\theta_{2}}{2}\right)\right. \\
& \left.+\frac{l^{2}}{r_{1} r_{2}} \sin \theta \sin \frac{3}{2}\left(\theta_{1}+\theta_{2}\right)\right\} .
\end{aligned}
$$

Figure 3 plots the non-dimensional ERR, $J / J_{0}$; that is, $K_{\mathrm{I}}^{2} / K_{\mathrm{I} 0}^{2}$ versus the non-dimensional slit length, $l / L_{0}$, for different combinations of $L_{0}$ and $t$, as calculated by Eq. (4). From these plots, it is clear that a slit longer than $1.2 L_{0}$ reduces $J / J_{0}$. Accordingly, a larger $l$ and a smaller $t$ results in a smaller $J$ (i.e., a larger reduction in ERR). In addition, a slit with a length of around $1.0 L_{0}$ occasionally results in a large $J / J_{0}$. This is simply because Eq. (3) gives a large $\sigma$ when $r_{1}$ is small. However, because the mutual interaction between a pre-crack and a slit of almost the same length has a large effect on SIF or ERR, the validity of Eq. (4) is not assured in this situation. Although some minor items remain to be verified, this simple analysis reveals that an appropriate slit reduces ERR in the bonding layer. This reduction should be big enough to prevent delamination of the composite from the metal mouthpiece.

${ }^{\dagger}$ Here, a solution for an internal crack in an infinite uniform plate is used instead of that for a surface crack in a semi-infinite plate.

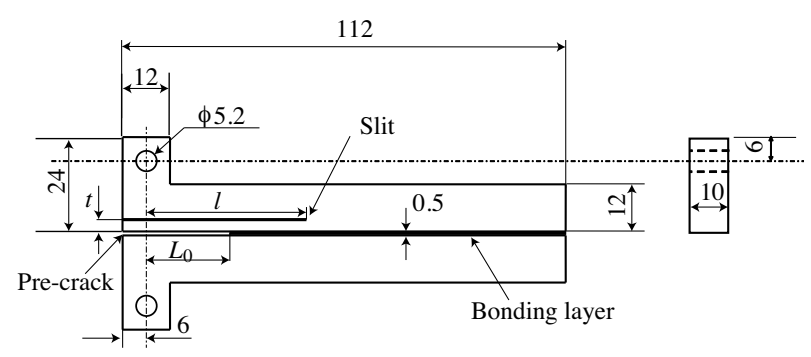

Fig. 4. Drawing of DCB test piece with slit $\left(L_{0}=24 \mathrm{~mm}, l=48 \mathrm{~mm}\right.$, $t=3 \mathrm{~mm})$.

\section{Experimental Verification}

\subsection{Test piece}

The effect of the slit was evaluated using simple DCB test pieces. Since the idea of the slit essentially can be used for metal/metal bonding as well as metal/composite bonding, it was decided to use symmetric DCB test pieces made of only metal parts.

Figure 4 shows a drawing of a DCB test piece. The test piece consisted of two C2801 brass parts bonded by Stycast 2850 FT with Catalyst 24 LV. During bonding, a kapton sheet was set on the bonding layer (at the left of Fig. 4) to put a pre-crack in the bonding structure. After curing, the kapton sheet was removed, and the location of the tip of the pre-crack was accurately tuned using a sharp cutting tool. After that, a slit was cut into the upper brass part by electrical-discharge machining. Since the slit was cut by electricaldischarge machining, the slit front had a round shape, whose radius was about $0.5 \mathrm{~mm}$ without any unwanted cracks. Typical pre-crack and slit dimensions were $L_{0}=24 \mathrm{~mm}$, $t=3 \mathrm{~mm}$ and $l=42 \mathrm{~mm}$ as shown in Fig. 4 . The breadth of the test pieces was uniformly $B=10 \mathrm{~mm}$. The DCB tests were carried out at room-temperature and a $0.01 \mathrm{~mm} / \mathrm{s}$ tension rate.

\subsection{Numerical verification}

A numerical verification using the finite element method (FEM) was carried out before the experiment. Figure 5 plots the values of the non-dimensional ERR, $J / J_{0}$ for a DCB test piece $\left(t / L_{0}=0.125\right)$. The figure also contains the experimental data presented in section 3.3 and the analytical solution of Eq. (4). The FEM results were based on the J-integral routine in Abaqus 6.10-3, ${ }^{10)}$ and each $J$ or $J_{0}$ value in the plot is the average of five paths of numerical J-integrations. The contact property was set on the crack surfaces, and interactions between the crack surfaces were prevented. All ERRs were calculated using the same process.

As shown in Fig. 5, the FEM results are less sensitive to changes in $l / L_{0}$ than the analytical ones. Especially around $l / L_{0}=1.0$, the FEM results do not become large, but rather stay around $J / J_{0} \leq 1.0$. It is speculated that this is because the pre-crack and slit of almost the same length interact and suppress any increase in $K_{\mathrm{I}}$.

In addition, the $J / J_{0}$ of the FEM increases slightly as $l / L_{0}$ increases for $l / L_{0} \geq 1.5$. It is speculated that this is because the finite ligament has a large effect on the DCB experiment. 


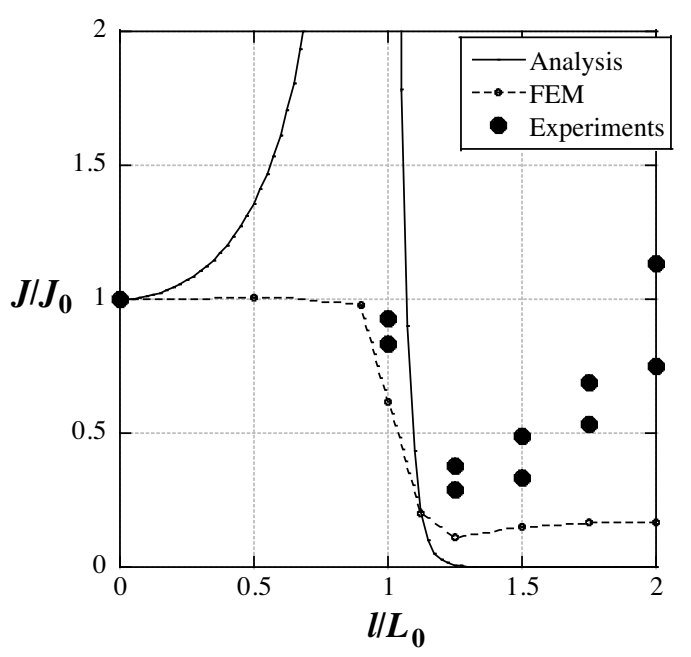

Fig. 5. Plot of non-dimensional ERR against non-dimensional slit length for analytical/FEM estimates and experimental results.

Summarizing the above, the analytical solution and FEM show decent agreement as far as their trends go, but they differ in their details.

\subsection{Experimental results}

Figure 5 also plots the experimental $J / J_{0}$ determined from the DCB test pieces with various slit lengths. In these tests, the crack growth occurred instantaneously, and it is difficult to evaluate the ERR using a nominal DCB method (for example, the modified compliance calibration method or ASTM D5528 for CFRP). Instead, $J / J_{0}$ was estimated as $F_{0}^{2} / F^{2}$, the square of the non-dimensional force at the moment crack growth starts, as follows.

Generally speaking, the ERR $J$ can be expressed in terms of the compliance $C$, crack length $a$, crack breadth $B$, and load $F$ as follows.

$$
J=\frac{F^{2}}{2 B} \frac{\mathrm{d} C}{\mathrm{~d} a}
$$

Here, $J / J_{0}$ means the ratio of ERR under the same conditions except for the presence of the slit. Accordingly, $J / J_{0}$ can be expressed as follows.

$$
\frac{J}{J_{0}}=\frac{\frac{F^{2}}{2 B} \frac{\mathrm{d} C}{\mathrm{~d} a}}{\frac{F^{2}}{2 B} \frac{\mathrm{d} C_{0}}{\mathrm{~d} a}}
$$

On the other hand, the force at the moment of crack growth is related with the critical ERR of the bonding layer as follows.

$$
J_{c}=\frac{F^{2}}{2 B} \frac{\mathrm{d} C}{\mathrm{~d} a}=\frac{F_{0}^{2}}{2 B} \frac{\mathrm{d} C_{0}}{\mathrm{~d} a}
$$

Substituting Eq. (7) into Eq. (6), we obtain

$$
\frac{J}{J_{0}}=\frac{\frac{\mathrm{d} C}{\mathrm{~d} a}}{\frac{\mathrm{d} C_{0}}{\mathrm{~d} a}}=\frac{F_{0}^{2}}{F^{2}} .
$$
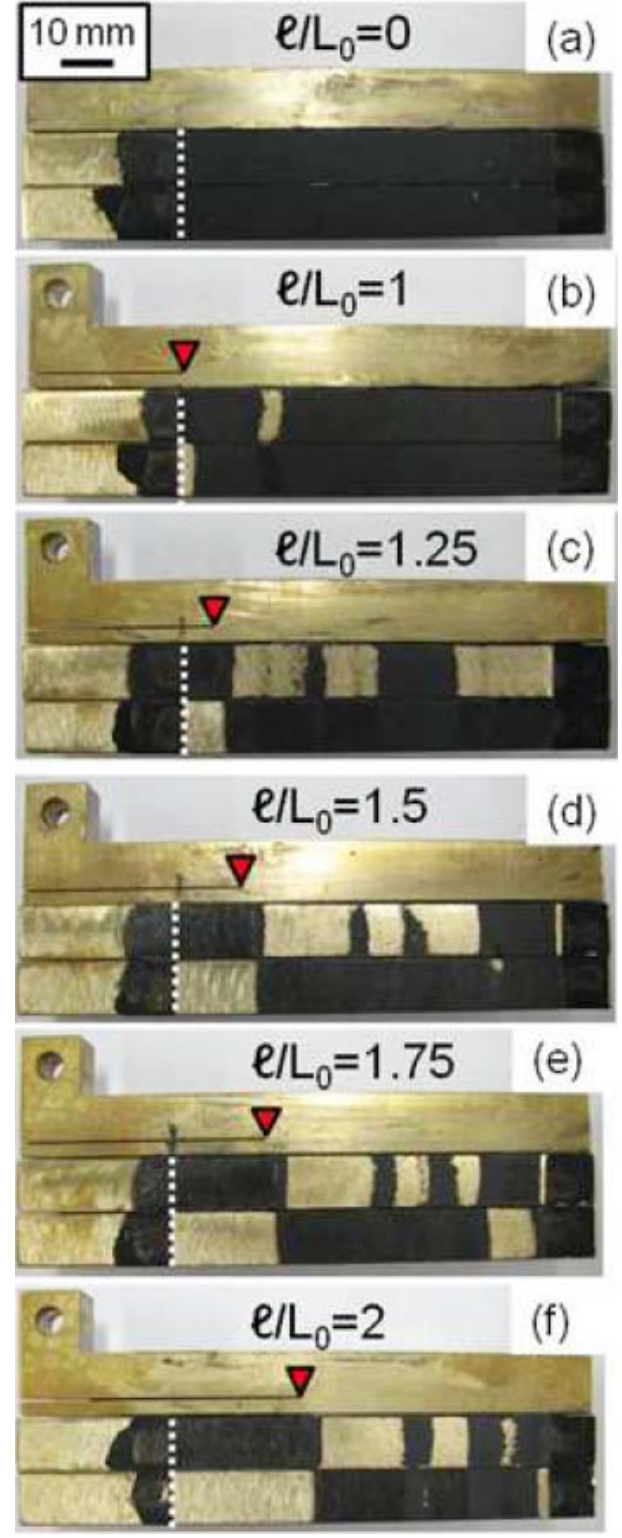

Fig. 6. Fracture surfaces of DCB test pieces.

Figure 6(a)-(f) shows the fracture surfaces of the bonded layer for various slit lengths. Each figure shows a side view of the upper part with the slit and the fracture surfaces of both sides. In each figure, the adhesive is in black, the white dotted line shows the position of the pre-crack tip, and the inverted triangle indicates the position of the slit tip. In Figs. 6(a) and (b), cohesive failure occurred over the entire bonded surface.

In Fig. 5, the experimental results for $l / L_{0} \leq 1.25$ show good agreement with the FEM results, but not with the simple analysis. $J / J_{0}$ is less than 1.0 , especially at $l / L_{0}=1.0$. On the other hand, for $l / L_{0}$ values larger than 1.5 , the experimental results deviate from the analytical and FEM results; they increase rapidly with increasing $l / L_{0}$. This reason is discussed in the next section.

\subsection{Discussion}

Figure 6(c)-(f) (i.e., results for a large $l / L_{0}$ ) shows some of the fracture surfaces. Generally, the appearance of a metal surface indicates the occurrence of interfacial debonding that 


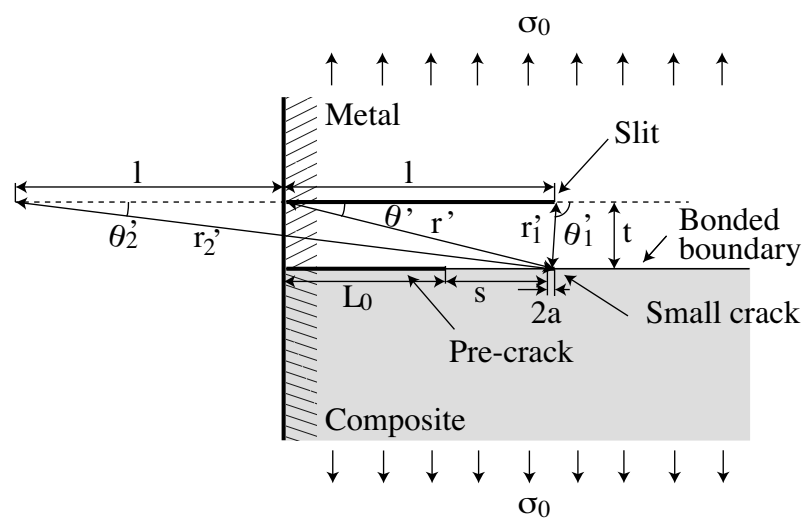

Fig. 7. Modified mathematical model of pre-crack, slit and diminutive internal crack in the bonding structure.

results in unstable fractures. The time histories of the crackgages in front of the pre-crack and slit indicate that the crack propagated from the slit-tip position toward the left in Fig. 6, but not from the pre-crack tip position toward the right. In addition, there was a strip of metal and adhesive just under the tip of the slit in the bonded interface. Therefore, it is considered that the fracture started not from the pre-crack tip but from the strip due to the effect of the slit-concentrated stress.

To evaluate this effect, the semi-infinite plate model of Fig. 2 was modified into the one shown in Fig. 7. A diminutive internal crack with a length equal to $2 a$ was added to the bonded boundary. When there is no pre-crack nor slit, the SIF of the diminutive internal crack tip, $K_{\mathrm{I} 0}^{\prime}$, can be expressed as

$$
K_{\mathrm{I} 0}^{\prime}=\sigma_{0} \sqrt{\pi a} .
$$

When there is no pre-crack nor diminutive internal crack, the stress due to the slit at the center of the diminutive internal crack $\sigma^{\prime}$ can be approximated as

$$
\begin{array}{r}
\sigma^{\prime}=\frac{\sigma_{0} r^{\prime}}{\sqrt{r_{1}^{\prime} r_{2}^{\prime}}}\left\{\cos \left(\theta^{\prime}-\frac{\theta_{1}^{\prime}+\theta_{2}^{\prime}}{2}\right)\right. \\
\left.+\frac{l^{2}}{r_{1}^{\prime} r_{2}^{\prime}} \sin \theta^{\prime} \sin \frac{3}{2}\left(\theta_{1}^{\prime}+\theta_{2}^{\prime}\right)\right\},
\end{array}
$$

where the variables $r^{\prime}, r_{1}^{\prime}, r_{2}^{\prime}, \theta^{\prime}, \theta_{1}^{\prime}$ and $\theta_{2}^{\prime}$ come from the geometrical configuration in Fig. 7.

Assuming that the effect of the pre-crack is negligible, because it is far enough from the diminutive internal crack, and that the slit increases the SIF at the diminutive internal crack tip $K_{\mathrm{I} 0}^{\prime}$ by a factor of $\sigma^{\prime} / \sigma_{0}$, the SIF at the diminutive internal crack tip when there is a slit $K_{\mathrm{I}}^{\prime}$ can be expressed as

$$
\begin{aligned}
K_{\mathrm{I}}^{\prime}= & \sigma^{\prime} \sqrt{\pi a} \\
= & \frac{\sigma_{0} r^{\prime} \sqrt{\pi a}}{\sqrt{r_{1}^{\prime} r_{2}^{\prime}}}\left\{\cos \left(\theta^{\prime}-\frac{\theta_{1}^{\prime}+\theta_{2}^{\prime}}{2}\right)\right. \\
& \left.+\frac{l^{2}}{r_{1}^{\prime} r_{2}^{\prime}} \sin \theta^{\prime} \sin \frac{3}{2}\left(\theta_{1}^{\prime}+\theta_{2}^{\prime}\right)\right\} .
\end{aligned}
$$

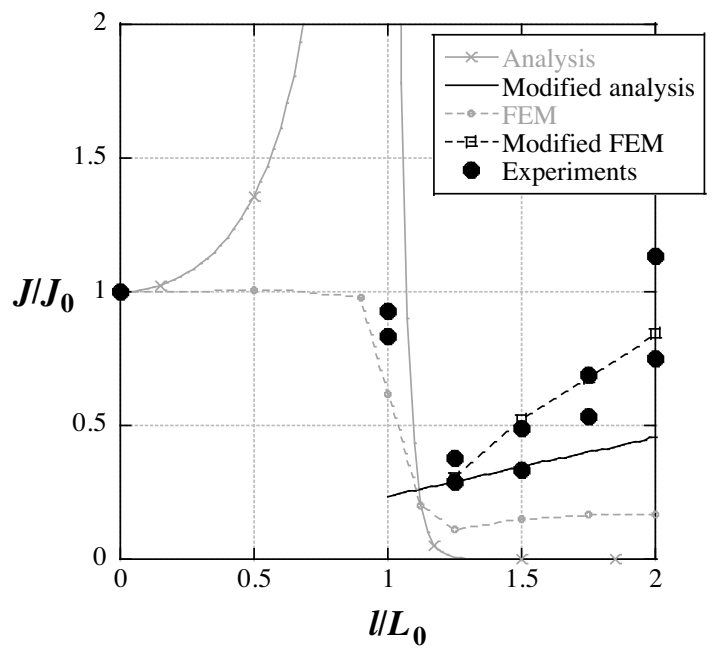

Fig. 8. Plot of non-dimensional ERR against non-dimensional slit length for modified analytical/FEM estimates and experimental results.

If $K_{\mathrm{I}}^{\prime}$ is larger than $K_{\mathrm{I}}$, the crack starts to grow from the diminutive internal crack rather than from the pre-crack. Here, $a$ is unknown, but it can be estimated to be $a=1.5 \mathrm{~mm}$ from the observed width of the adhesive strips on the fracture surfaces; the width is nearly constant for the examined conditions (Fig. 6(c)-(f)). Although $s$ is also unknown, it was arbitrarily taken to be the value that gives the largest $K_{\mathrm{I}}^{\prime}$.

The new estimate, $J^{\prime} / J_{0}=K_{\mathrm{I}}^{\prime 2} / K_{\mathrm{I} 0}^{2}$, based on the modified model is plotted in Fig. 8 as "Modified analysis," and the FEM results simulating the diminutive internal crack of length $2 a$ under the tip of the slit are plotted as "Modified FEM." The trends of these lines are in agreement with the experimental results. Thus, it can be concluded that the stress concentration around the slit-tip affects the bonded boundary, causes a diminutive internal crack, and increases the actual ERR. It must be pointed out that the details of interfacial debonding to make a diminutive internal crack inside of the bonded layer remain unclear. However, it is very important to choose appropriate values of $t$ and $l$ in relation to $L_{0}$.

\subsection{Guidelines}

Basic guidelines were derived for choosing $t$ and $l$ by referring to the plots of non-dimensional ERR based on Eqs. (4) and (11) for various $t$ (see Fig. 9). The length of the diminutive internal crack $2 a$ was set as $3.0 \mathrm{~mm}$ after referring to the DCB results. Solid lines in the figure indicate the trends of the non-dimensional ERR, taken to be the larger value of Eqs. (4) and (11).

The overall trend in Fig. 9 indicates that a small $t$ gives a good reduction in ERR at the pre-crack tip, but causes a large growth in ERR at the diminutive internal crack. Therefore, a moderately large $t$ and $l$ are better and can be used as a guideline. In addition, cohesive failure is strongly desired in order to prevent an unstable fracture. Thus, a $t$ of $1.0 L_{0}$ and $l$ of $1.8 L_{0}$, which reduce the ERR to a tenth of its value when there is no slit, are recommended, although the choice depends on the actual boundary conditions. 


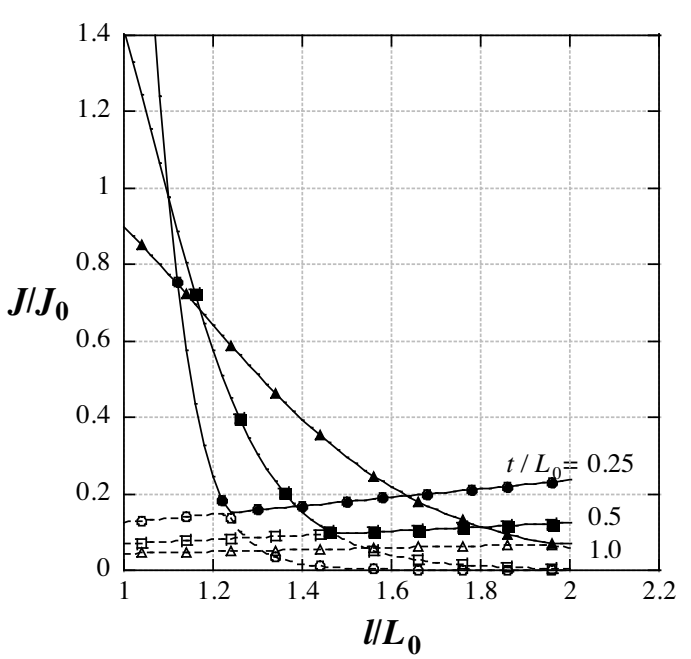

Fig. 9. Change in profile of non-dimensional ERR against non-dimensional slit length for various $t$.

\begin{tabular}{lc} 
Table 1. Properties of target pressure vessel. \\
\hline Diameter & $700 \mathrm{~mm}$ \\
Length & $1,200 \mathrm{~mm}$ \\
MEOP & $13.5 \mathrm{MPa}$ \\
Proof pressure & $22.5 \mathrm{MPa}$ \\
Burst pressure & $27.0 \mathrm{MPa}$ \\
\hline
\end{tabular}

\section{Example of Pressure Vessel Design}

\subsection{Pressure vessel}

This section presents an example of a pressure vessel's design satisfying the strength constraints, but not the fracture mechanical constraint. The addition of a slit improves the pressure vessel design.

The vessel is intended to be a high-pressure gas tank. It is a polymer-lined composite tank produced by filament winding (FW). The properties of the tank are listed in Table 1.

Figure 10 shows the pressure vessel's mesh for the FEM simulation. The vessel is axisymmetrical, and the figure is a cross-section of the upper half of the vessel. The lower half is symmetrical to the upper one and a plane symmetrical boundary condition is set on the boundary nodes. Axisymmetric solid elements are used for this model.

The light gray part of the mesh is the mouthpiece made of titanium alloy, and the dark gray part is CFRP made by FW. The CFRP layer and titanium mouthpiece are directly bonded by resin in the CFRP prepregnation process. A surface pre-crack is located in the bonded boundary between the titanium and composite. The vessel has an additional GFRP layer for production reasons. It is bonded by film adhesive under the CFRP layer from the equator to the shoulder. Figure 10 shows the laminated constitution around the edge of the mouthpiece. The GFRP layer and the film adhesive are modeled as actual elements in the mesh.

The properties of the materials used in the FEM are shown in Table 2. In our design policy for this vessel, plastic deformation of titanium alloy is allowed above the maximum ex-

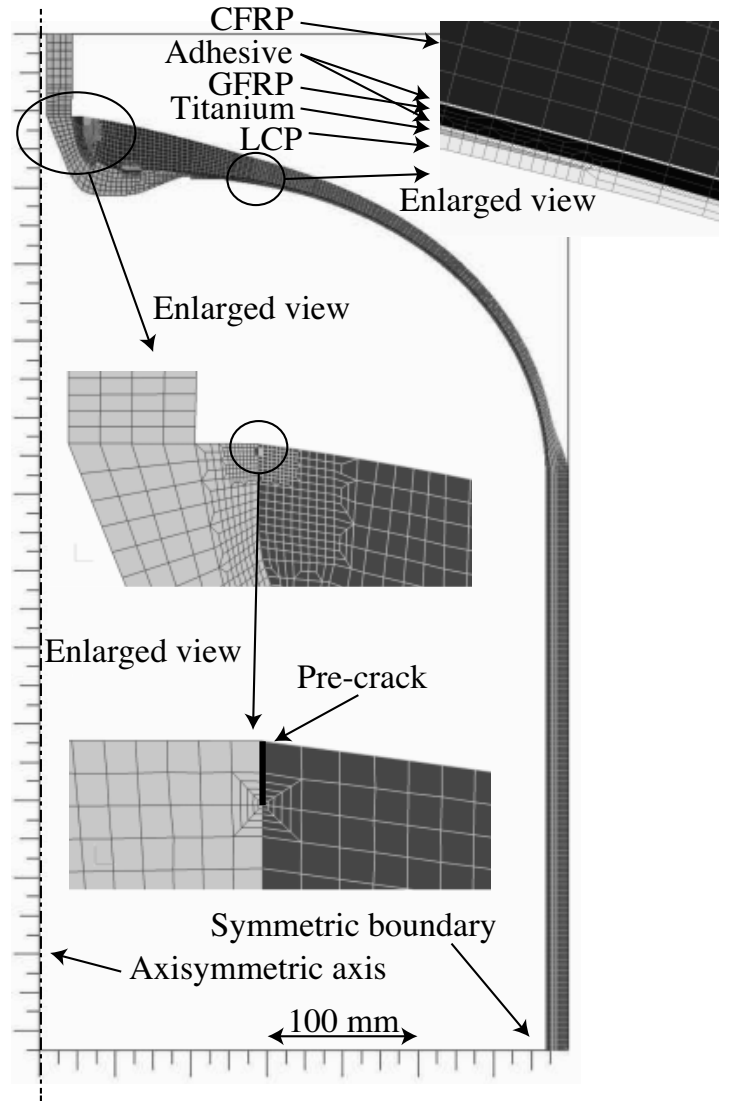

Fig. 10. FEM mesh of example pressure vessel.

Table 2. Material properties of target pressure vessel.

\begin{tabular}{ccc}
\hline Part & Description & $\begin{array}{c}\text { Young's modulus } \\
E(\mathrm{GPa})\end{array}$ \\
Material & Fiber direction & 174. \\
Composite & Fiber normal & 10.0 \\
CFRP & above 910 MPa & 116. \\
\hline Mouthpiece & 12.3 \\
Ti-6Al-4V & & 20.7 \\
\hline Liner reinforcer & Pseude-isotropic & 4.00 \\
GFRP & Isotropic & 1.50 \\
\hline Liner &
\end{tabular}

pected operation pressure (MEOP). The yield stress of titanium alloy is $910 \mathrm{MPa}$ in von Mises stress and the plastic behavior is modeled with a bi-linear and kinematic hardening model.

\subsection{Improvements due to slit}

The FEM calculation reveals that this vessel satisfies the strength requirements except for the stress concentration around the pre-crack front (i.e., the maximum/minimum stresses and strains are within the limits in the absence of the pre-crack between CFRP and metal mouthpiece), though a plastic region arises in the metal mouthpiece at burst pressure (there is no plastic region at MEOP). Here, let us consider the stress concentration around the pre-crack from the viewpoint of fracture mechanics. 
Radial stress (MPa)

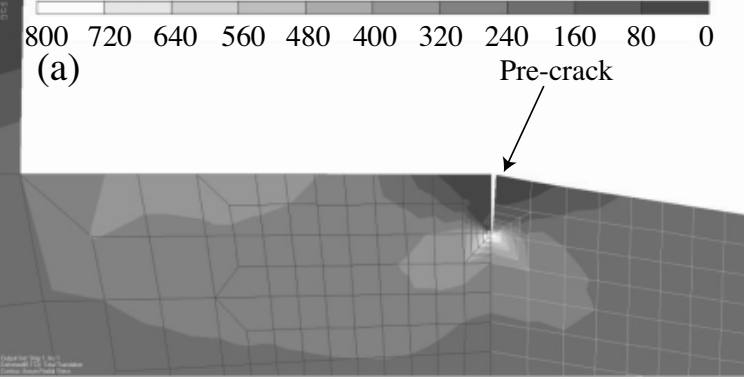

(b)

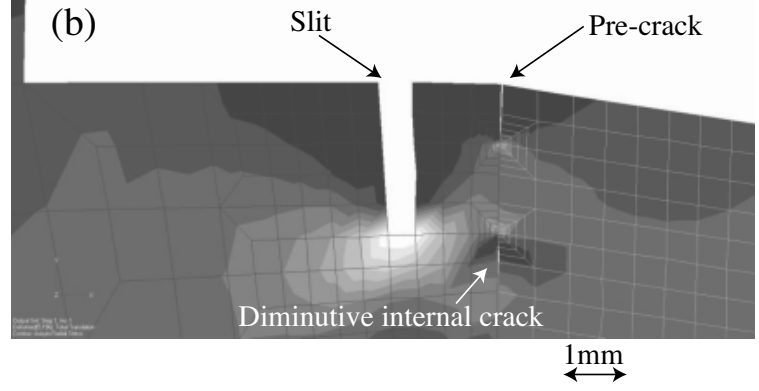

Fig. 11. Radial stress around pre-crack: (a) without slit and (b) with slit.

Figure 11 shows the distribution of radial stress at burst pressure in the region corresponding to Part A in Fig. 1. The stress works to open the pre-crack. When the surface pre-crack length is $1 \mathrm{~mm}\left(=L_{0}\right)^{\ddagger}$ and there is no slit (see Fig. 11(a)), the ERR at the tip of the pre-crack becomes $14,500 \mathrm{~J} / \mathrm{m}^{2}$ in the FEM at burst pressure. On the other hand, the critical ERR of the titanium/CFRP bonding was measured to be $1,420 \mathrm{~J} / \mathrm{m}^{2}$ in previous DCB tests. As this critical value was measured in the DCB tests, it is the value for mode-1 crack propagation, not the exact value for a mixed mode of mode- 1 and mode- 2 . Although the above FEM estimations give the ERR for a mixed-mode (i.e., sum of mode- 1 and mode-2), the critical value for mode- 2 is supposed to be larger than that of mode-1, and the comparison of the ERR estimated for the mixed mode with the critical ERR for mode-1 is supposed to be conservative in general.

In the above evaluation, the pre-crack has a cylindrical shape because of the axisymmetrical elements, and the evaluation is conservative. Now let's consider a realistic semi-elliptical-shaped surface crack with length and width of $1 \mathrm{~mm}$ $\left(L_{0}\right)$ and $1.67 \mathrm{~mm}\left(L_{0} / 0.6\right)$, respectively. According to Newman and Raju, ${ }^{11)}$ the dimensionless SIF at the semi-elliptical surface crack with the ligament of $4 L_{0}$ is estimated to be 1.110. On the other hand, that of the through-surface crack with the same ligament is 1.372 . Therefore, the semi-elliptical crack has an ERR approximately 0.6545 that of the through-crack. Supposing these values can be applied to a cylindrical crack and the semi-elliptical crack, the above ERR at the pre-crack tip falls to $9,490 \mathrm{~J} / \mathrm{m}^{2}$, but this value still exceeds the critical ERR. Thus, this vessel does not sat-

*This value was chosen after considering the detection capability of nondestructive inspection.

${ }^{\S}$ Typical fatigue strength of Ti-6Al-4V for $10^{7}$ cycles is about 500-700 MPa.
Table 3. Summary of ERRs in the target pressure vessel of Part A.

\begin{tabular}{|c|c|c|c|}
\hline & Crack shape & Cylindrical & Elliptical \\
\hline Condition & Position & $\operatorname{ERR}\left(\mathrm{J} / \mathrm{m}^{2}\right)$ & $\operatorname{ERR}\left(\mathrm{J} / \mathrm{m}^{2}\right)$ \\
\hline Without slit & Pre-crack & 14,500 & 9,490 \\
\hline \multirow[t]{2}{*}{ With slit } & Pre-crack & 1,700 & 1,110 \\
\hline & Internal crack & 2,140 & 1,400 \\
\hline Critical ERR & $\mathrm{N} / \mathrm{A}$ & 1,420 & 1,420 \\
\hline
\end{tabular}

isfy the fracture mechanical constraint.

To solve this fracture mechanical problem, let us add a slit to the titanium mouthpiece. According to the guidelines in section 3.5, and after some FEM trials, values of $t=$ $1.5 \mathrm{~mm}=1.5 L_{0}$ and $l=2.5 \mathrm{~mm}=2.5 L_{0}$ were chosen. Figure 11(b) shows the distribution of radial stress at burst pressure for a vessel with a slit. A diminutive internal crack with a length equal to $L_{0}$ near the slit tip is also considered. Although the front of the slit is square in the FEM because of difficulty in re-meshing, the shape should be round in order to suppress stress concentration in actual tanks. Such a slit causes a moderate stress increase around the round front, about three times larger than the value without a slit, and the stress reaches about $800 \mathrm{MPa}$ at burst pressure. The stress in nominal operation is half of the stress at burst pressure, about $400 \mathrm{MPa}$, and this value is small enough to prevent fatigue crack propagation. ${ }^{\S}$

The results of the FEM calculation revealed that the radial stress at the cylindrical pre-crack tip relaxes, and the ERR drops to $1,700 \mathrm{~J} / \mathrm{m}^{2}$. The ERR at a cylindrical diminutive internal crack tip is $2,140 \mathrm{~J} / \mathrm{m}^{2}$. In a more realistic case, the ERRs at the semi-elliptical pre-crack and the elliptical diminutive internal crack are estimated to be 1,110 and $1,400 \mathrm{~J} / \mathrm{m}^{2}$, respectively. These values are summarized in Table 3. Both values satisfy the critical ERR of the bonding boundary. Thus, it is concluded that this pressure vessel with a slit satisfies not only the strength constraint, but also the fracture mechanical constraint.

\section{Conclusions}

This study dealt with the fracture mechanical problem of large high-pressure vessels (i.e., delamination of the bonding layer between the composite and metal), especially around the mouthpiece. A simple method in which a slit is put in the mouthpiece near the bonding layer was shown to reduce the energy-release rate to less than one-tenth of its original value in an analytical study of a semi-infinite plate. The effect of the slit was then experimentally studied for slits of different lengths and positions in double cantilever beam tests and finite element method calculations. Finally, an example of pressure vessel design showing the improvement by adding the slit was presented.

\section{References}

1) Composites for Cryotank Structures. Present and Future, George C. Marshall Space Flight Center, 1996. 
2) Goetz, R. C., Ryan, R. S., and Whitaker, A. F.: Final Report of the X-33 Liquid Hydrogen Tank Test Investigation Team, George C. Marshall Space Flight Center, 2000.

3) Johonson, T., Sleight, D., and Martin, R.: Structures and Design Phase I Summary for the NASA Composite Cryotank Technology Demonstration Project, AIAA Paper 2013-1825, 54th AIAA/ASME/ ASCE/AHS/ASC Structures, Structural Dynamics, and Materials Conference, 2013.

4) NASA Completes Successful Battery Tests on Composite Cryotank NASA, http://www.nasa.gov/press/2014/august/nasa-completessuccessful-battery-of-tests-on-composite-cryotank/index.html

5) Higuchi, K., Takeuchi, S., Sato, E., Naruo, Y., Inatani, Y., Namiki, F., Tanaka, K., and Watabe, Y.: Development and Flight Test of Metallined CFRP Cryogenic Tank for Reusable Rocket, Acta Astronautica, 57 (2005), pp. 432-437.

6) Takeuchi, S., Sato, E., Naruo, Y., Nonaka, S., Arakawa, Y., and Miyahara, A.: Design and Development of Polymer-lined Composite Tank for Liquid Hydrogen Tank of Reusable Vehicle Test, 59th International Astronautical Congress IAC-08-C2.1.5, 2008.
7) Takeuchi, S., Sato, E., Onoda, J., Higuchi, K., and Arakawa, Y.: Evaluation of Adhesive Bonding Structure in Cryogenic Composite Tank Based on Fracture Mechanics, T. Jpn. Soc. Aeronaut. Space Sci., 52 (2009), pp. 36-46.

8) Benton, J., Ballinger, I., Ferretti, A., and Ierardo, N.: Design and Manufacture of a High Performance, High Mass Efficient Gas Tank for the VEGA AVUM, AIAA Paper 2007-5500, 43rd AIAA/ASME/SAE/ ASEE Joint Propulsion Conference, 2007.

9) Takeuchi, S. and Sato, E.: Bonding Structure of Metal Member and Composite-material Member, WO 2011/052714 A1.

10) Shih, C. F., Moran, B., and Nakamura, T.: Energy Release Rate along a Three-Dimensional Crack Front in a Thermal Stressed Body, Int. J. Fracture, 30 (1986), pp. 79-102.

11) Newman, J. C. Jr. and Raju, I. S.: Analysis of Surface Cracks in Finite Plates under Tension or Bending Loads, NASA TP 1578, 1979.

C. Hwu

Associate Editor 